Published in final edited form as:

Int Psychogeriatr. 2010 March ; 22(2): 281-290. doi:10.1017/S1041610209991001.

\title{
Trajectories of Cognitive Decline in Alzheimer's Disease
}

\author{
Patricia A. Wilkosz ${ }^{1}$, Howard J. Seltman ${ }^{3}$, B. Devlin ${ }^{1}$, Elise A. Weamer ${ }^{1,2}$, Oscar L. \\ Lopez $^{2,1}$, Steven T. DeKosky 2,1 , and Robert A. Sweet ${ }^{1,2,4}$ \\ ${ }^{1}$ Department of Psychiatry, University of Pittsburgh, Pittsburgh, PA \\ 2 Department of Neurology, University of Pittsburgh, Pittsburgh, PA \\ ${ }^{3}$ Department of Statistics, Carnegie Mellon University, Pittsburgh, PA \\ ${ }^{4}$ VISN 4 Mental IIIness Research, Education and Clinical Center (MIRECC), VA Pittsburgh \\ Healthcare System, Pittsburgh, PA
}

\section{Abstract}

Background-Late-onset Alzheimer disease (LOAD) is a clinically heterogeneous complex disease defined by progressively disabling cognitive impairment. Psychotic symptoms which affect approximately one-half of LOAD subjects have been associated with more rapid cognitive decline. However, the variety of cognitive trajectories in LOAD, and their correlates have not been well defined. We therefore used latent class modeling to characterize trajectories of cognitive and behavioral decline in a cohort of AD subjects.

Methods-201 Caucasian subjects with possible or probable AD were evaluated for cognitive and psychotic symptoms at regular intervals for up to 13.5 years. Cognitive symptoms were evaluated serially with the Mini-Mental State Examination (MMSE), and psychotic symptoms were rated using the CERAD behavioral rating scale (CBRS). Analyses undertaken were latent class mixture models of quadratic trajectories including a random intercept, with initial MMSE score, age, gender, education, and APOE $\varepsilon 4$ count modeled as concomitant variables. In a secondary analysis, psychosis status was also included.

Results-AD subjects showed six trajectories with significantly different courses and rates of cognitive decline. The concomitant variables included in the best latent class trajectory model were initial MMSE and age. Greater burden of psychotic symptoms increased the probability of following a trajectory of more rapid cognitive decline in all age and initial MMSE groups. APOE $\varepsilon 4$ was not associated with any trajectory.

For questions and correspondence please contact: Robert A. Sweet, M.D., Professor of Psychiatry and Neurology, Vice-Chair, Institutional Review Board, University of Pittsburgh., Co-Associate Director for Research, Mental Illness Research, Education, and Clinical Center, VA Pittsburgh Healthcare System., Ph +1 412383 8548, Fax +1 412624 9910, sweetra @ upmc.edu, http://www.wpic.pitt.edu/research/sweetlab. Mail: Biomedical Science Tower, Rm W-1645, 3811 O'Hara Street, Pittsburgh, PA 15213-2593. Express Mail: Biomedical Science Tower, Rm W-1645, Lothrop and Terrace Streets, Pittsburgh, PA 15213-2593.

\section{DESCRIPTION OF AUTHORS' ROLES}

Dr. Patricia Wilkosz was the primary author of this manuscript. Dr. Howard Seltman contributed to the design of the study, conducted the statistical analysis of the data and assisted in the writing of the manuscript. Dr. Bernard Devlin contributed to the design and analysis of the study. Drs. Wilkosz, Lopez, DeKosky, and Sweet, and Ms. Weamer contributed to the recruitment and diagnoses of patients, and data collection at the Alzheimer Disease Research Clinic. Dr. Sweet contributed to the study design and writing of the manuscript. In addition, all authors reviewed drafts of the manuscript.

\section{CONFLICT OF INTEREST DECLARATION}

Sources of Financial Support: Supported in part by research grants AG027224 and AG005133 from the National Institute of Aging. Information about Financial Relationships: The authors do not have a financial relationship with any organization that might have vested interest in the conduct and reporting of the study. 
Conclusion-Trajectory modeling of longitudinal cognitive and behavioral data may provide enhanced resolution of phenotypic variation in Alzheimer disease.

\section{Keywords}

Late-onset Alzheimer disease (LOAD); Psychosis; Cognitive neuropsychology in dementia; Assessment of cognitive disorders/dementia; APOE $\varepsilon 4$

\section{INTRODUCTION}

Late-onset Alzheimer disease (LOAD) is a clinically heterogeneous complex disease presumed to result from the interaction of multiple genetic and environmental risk factors (Holmans et al., 2005;Bertram et al., 2007). It is defined by progressively disabling cognitive impairment. LOAD is also associated with frequent behavioral symptoms, such as psychosis, that are associated with greater cognitive (Ropacki and Jeste, 2005) and functional (Scarmeas et al., 2005) decline. Longitudinal symptom course has not been clearly delineated in LOAD, and this contributes significantly to phenotypic heterogeneity (Healy, 2006; Tyas et al., 2007). Questions remain regarding the natural history of disease factors affecting rate of decline, and whether $\mathrm{AD}$ is a single disease or a heterogeneous combination of closely related disorders with varying patterns of cognitive and behavioral decline.

The goals of most longitudinal studies involving signs and symptoms of LOAD include characterization of individual patterns of change (trajectories), how these patterns vary according to demographic, clinical and/or genetic characteristics, and how much individuals with the same characteristics vary from one another. Longitudinal studies have many advantages over cross-sectional studies, but are more complex analytically. A variety of analytical approaches have been used for such tasks, including two-stage analysis (Mann et al., 1992), hierarchical (mixed) models (Rasmusson et al., 1996), and structural equation (latent growth curve) models (Royall et al., 2005). These methods tend to minimize or obscure the fact that similar subjects may follow quite different trajectories.

Latent class trajectory models have been applied successfully in a variety of longitudinal studies in psychiatry in recent years (Curran and Willoughby, 2003), and offer several methodological advantages. These include the grouping of similar individual trajectories into interpretable classes, which may then be used to identify additional underlying genetic and/or environmental causes of differences in disease trajectories. Recent examples of latent class trajectory models include psychological adjustment to breast cancer (Helgeson et al., 2004) and functional changes in non-demented elderly (Dodge et al., 2006). These models, not be confused with latent growth curve models, are usually formulated as an extension of mixed models, in which a given subject may follow a weighted mixture of several entirely different trajectories. The weights represent a set of probabilities, one for each trajectory. In some cases, many subjects have most of their probability focused on a single trajectory; in which case the trajectories can be thought of as potential paths that different subjects can follow. It is here that the term "latent class" is most appropriate: the trajectories represent different classes of subjects whose existence is inferred from the patterns in all of the data. In other cases, subjects' probabilities include two or more trajectories with substantial probability. In the latter instance, the model trajectories are better thought of as a mathematical approach to modeling heterogeneity beyond what can be well characterized by mixed models (Jones et al., 2001), and careful thought needs to go into interpretation of results.

We sought to use latent class modeling to characterize the trajectories of cognitive decline, including the effects of demographic and clinical covariates, in a cohort of 201 patients with a final diagnosis of possible/probable $\mathrm{AD}$. We report findings from subjects evaluated for 
cognitive and behavioral (psychosis) symptoms at regular intervals of up to 13.5 years. The analysis showed that divergent trajectories could be defined, enhancing phenotypic resolution. Among other possibilities, differing trajectories may reflect the distinct contributions of underlying pathologies (e.g. amyloid, tau, vascular, and Lewy body) and/or of specific genetic variants.

\section{METHODS}

\section{Subjects}

201 Caucasian subjects with a final diagnosis of possible or probable AD were evaluated at the University of Pittsburgh Alzheimer Disease Research Center (ADRC), Pittsburgh, PA, between 1987 and 2007. Demographic information was recorded at the initial visit. Complete medical and neuropsychiatric histories and examinations were conducted, and diagnoses recorded. A comprehensive neuropsychological test battery was administered to all subjects as previously described (Lopez et al., 2003). Following evaluation, subjects were diagnosed with possible or probable AD using criteria of the National Institute of Neurological and Communicative Disorders and Stroke-Alzheimer Disease and Related Disorders Association (NINCDS-ADRDA) (McKhann et al., 1984). Diagnostic assessments including interim histories, neurological exams, and neuropsychological testing were repeated annually during clinic visits and used to confirm or revise clinical diagnoses as needed. Patients with schizophrenia or bipolar disorder using (DSM-IV) criteria, or probable Dementia with Lewy Bodies (McKeith et al., 1996) at their initial or follow-up assessments were excluded. The rate of autopsy confirmation of AD in subjects diagnosed with Possible or Probable AD exceeds $90 \%$ in our center (Lopez et al., 2003). All data collected in this study were obtained with protocols approved by the Institutional Review Board of the University of Pittsburgh.

\section{Assessment of Cognition}

Cognition was evaluated serially with the Mini Mental State Examination (MMSE) (Folstein et al., 1975). This global test of cognitive function was administered to subjects at least annually at clinic visits. Subjects with fewer than four MMSE scores (to allow fitting of a quadratic curve) were excluded,

\section{Assessment of Psychosis}

Psychosis was evaluated with the CERAD behavioral rating scale (CBRS) (Tariot et al., 1995). The CBRS measures the extent of behavioral pathology in persons with dementia, and was designed to be administered to an informant. Psychosis was defined by the presence of persistent hallucinations or delusions occurring at any time during the course of the dementia. A delusion was defined as a false belief based on incorrect inference about external reality, resistant to persuasion or contrary evidence, and not attributable to social or cultural mores. Hallucinations were defined as sensory perceptions for which there was no basis in reality. Discrete hypnogogic and hypnopompic hallucinations, as well as symptoms occurring only during an episode of delirium, were not rated. Inter-rater reliability for the determination of psychosis using the CBRS at the ADRC has been established and is uniformly high, with mean (SD) AD with psychosis kappa 0.98 (0.08) for all raters and mean (SD) individual psychosis symptom kappas ranging from $0.98(0.08)$ to $1.0(0.0)$. The CBRS was administered at initial and annual visits.

\section{Genotyping}

DNA for determination of apolipoprotein E genotype was isolated with the QIAmp kit from Qiagen (Chatsworth, CA). Genotyping was performed using an established PCR protocol previously described (Kamboh et al., 1995). 


\section{Statistical Analysis}

The analyses undertaken were latent class mixture models of quadratic trajectories including a random intercept and concomitant variables. Models were fit using flexmix package (Leisch, 2004) of the statistical program R, version 2.8.1, from the R Foundation for Statistical Computing (R Development Core Team, 2007). In the analyses, MMSE scores at entry and each return visit were modeled as a continuous outcome measure. Psychosis status was defined by the presence of persistent hallucinations or delusions, operationalized for the CBRS as a score of 2, 3, or 4. Psychosis burden was defined using the maximum (per visit) sum of the psychosis items as scored on the CBRS: $0=$ Never occurred; $1=1-2$ days in past month; $2=3-$ 8 days in past month; $3=9-15$ days in past month; $4=>16$ days in past month. Models used either psychosis burden or psychosis status as concomitant variables.

Data were modeled by mixed models without the latent class component and with latent class trajectory models. Both types of models were built with generalized linear models using a quadratic function of time, and a random intercept for the group trajectories. The mixed models also used a random slope, and the latent class trajectory models used multinomial logit models for the concomitant variables to define the probabilities that a given subject will be grouped in a particular trajectory. Initial MMSE score, age, gender, education, psychosis status or burden, and APOE $\varepsilon 4$ count were modeled as concomitant variables in the latent class trajectory models and as independent variables (with and without interaction with time) in the mixed models. Model parameters were estimated with the Expectation-Maximization (EM) algorithm, which finds the (local) maximum likelihood parameters for each model. Because latent class models may have local maxima, we used 20 random starting values for each model, which we found to have a very high chance of finding the global maximum. In addition for each set of concomitant variables tested, we ran models with 1 through 8 latent trajectories, and chose the best fitting model. Relative goodness-of-fit was assessed using Bayesian Information Criteria (BIC) (Schwarz, 1978).

For analyses including APOE $\varepsilon 4$ we dropped the one subject with missing data. For both the mixed models and the latent class trajectory models, missing observations in years need no special handling because time is modeled explicitly (not visit number) and because both models are unbiased for missing at random data.

\section{RESULTS}

\section{Subject Characteristics}

Demographic and clinical characteristics of our subjects are presented (Table 1). The average baseline age of individuals in the study was 73.3 years. Slightly over half were female (59.2\%), and the average number of years of education was 13.2. Most subjects carried a diagnosis of Probable AD (92.5\%), with the remainder classified as Possible AD (7.5\%). Most participants demonstrated mild to moderate impairment at presentation as reflected by a mean baseline MMSE score of 21.0. Approximately half of all subjects (58.2\%) exhibited psychotic behavior during follow-up, consistent with other reports. Mean duration of follow-up was 53.1 months, resulting in a total of 1500 visits for available for mixed and trajectory modeling in the 201 subjects. Baseline age decreased as the number of APOE $\varepsilon 4$ alleles increased (median ages for 0 to 2 alleles are 77,74 and 72 years; Kruskal-Wallis $\chi^{2}=7.78, p=0.02$, data not shown).

\section{Mixed Modeling of Cognitive Course}

The results from the best mixed model using backward stepwise selection from a model with all interactions are shown in table 2. The standard deviation of the random intercept is 0.90 and the random slope is 1.32 . 


\section{Trajectory Modeling of Cognitive Course}

Latent class models were fit using linear, quadratic, and cubic curves over time, with a range of 1 to 8 latent classes, and with no concomitant variables up to first order interactions of age, gender, education, APOE $\varepsilon 4$ status, and excluding and including psychosis status. BIC was used to choose the best models. A quadratic trajectory term was justified, but a cubic term did not improve the BIC. The best model had six trajectories (latent classes or "groups", Figure 1). Although visual inspection of Figure 1 might suggest that the three poorer and the three better trajectories could be combined to simplify the model to only two trajectories, we found the six group model $(\mathrm{BIC}=5510)$ to be to strongly favored over the two group model (BIC=5986). The parameters of the 6 trajectories for the best-fitting model excluding psychosis status are shown in Table 3.

The concomitant variables included in the best latent class trajectory model are initial MMSE and age. Because the interpretation of concomitant variable parameters is difficult, we present the effects of initial MMSE and age on the probabilities of subjects falling into the different trajectories graphically. Figure 1, at top, shows the individual cognitive trajectories for the latent class model with the best fit to the data, the 6-group quadratic trajectory model with initial MMSE and age as concomitant variables. When gender, education and apolipoprotein E genotype were included in the model, there was no improvement in the BIC. Each row of the bar graph in the lower half of the figure shows the probability distribution across trajectories for some specific values of the concomitant variables. The colors in the bar graphs refer the correspondingly colored trajectories. For example, the top line of the bar graph represents 68 year old subjects with an initial MMSE of 26. We can read the approximate probabilities of falling into trajectories 1,2 and 4 as 50,20 and 30\% respectively, with almost no chance of falling along any of the other trajectories.

Because latent class analysis models each subject's trajectory as a mixture of all trajectories, it is worth examining how often subjects fit predominantly into one particular trajectory vs. a mixture of two or more. For our models, $66 \%$ of subjects had at least $95 \%$ of their probability focused on a single trajectory, and $89 \%$ of subjects had at least $95 \%$ of their probabilities focused on just two trajectories. Of the remaining 22 subjects, the majority (63.6\%) had a sum of at least $90 \%$ for the top two probabilities. This suggests that six trajectories well represent the patterns of MMSE change for the majority of our subjects.

\section{Trajectory Modeling of Cognitive and Behavioral Course}

Figure 2 shows cognitive trajectories of AD subjects with psychosis modeled as a covariate. This model showed the same BIC as the above model, but some of the concomitant parameters were statistically significant. The bottom panel of Figure 2 shows the probabilities of each subject belonging to a particular trajectory, as a function of initial MMSE score, age and psychosis burden. These results demonstrate that AD subjects of all ages and initial MMSE scores who develop psychosis had an increased likelihood of following a more rapidly declining MMSE curve (trajectories 3, 5 and 6). Younger subjects who developed psychosis and presented with low MMSE score were more likely to be in the groups with most rapidly declining MMSE (groups 5 and 6).

Using a probability cutoff of 0.65 , we classified approximately $92 \%$ (186 subjects classified out of 201) of the subjects. Table 4 shows the covariate distribution per trajectory group. The range in group size was $24-35$, with approximately equal numbers in each group. The youngest subjects (60-70) are more likely to be in group 6, and show the most rapid cognitive decline. Males predominate over females only in trajectory 1 . Subjects in trajectories 1 through 3 had the most education. The fraction of subjects who develop psychosis varied from $29 \%$ in group 1 to $86 \%$ in group 5 . A similar trend of psychotic subjects showing more rapid cognitive decline 
is also seen with psychosis burden, with a mean psychosis score of 1.79 in group 1 to 731 in group 5.

\section{DISCUSSION}

These models from a longitudinal cohort of AD subjects show six trajectories with significantly different courses and rates of cognitive decline. These trajectories were best defined by subject age and MMSE at the initial assessment. In general, subjects with older age followed a trajectory with slower cognitive decline Subjects with higher initial MMSE scores tended to follow trajectories of slower decline, while those with lower initial scores tended to decline more rapidly. On the other hand, the latent trajectory modeling makes clear that a substantial minority of subjects with high initial MMSE score follow a poorer trajectory (trajectory 3), while a substantial minority of those with low initial scores do not decline rapidly (trajectory 4). Within each age and initial MMSE group, subjects with psychosis follow a course of more rapid decline.

Importantly the latent class trajectory model demonstrates heterogeneity of individuals more effectively than mixed models. One of the key features of latent class trajectory models is that in addition to identification of the latent trajectories, they also allow modeling of so-called concomitant variables. Concomitant variables are categorical or quantitative subject characteristics which help to characterize the probabilities of falling into the various latent trajectories. Technically, a concomitant model is based on the multinomial logit model (Gruen and Leisch, 2008). This is a major distinguishing feature between latent class trajectory models and mixed trajectory models. Mixed trajectory models assume that the individual trajectories for all subjects with a given set of measured explanatory variables follow a Normal distribution around a mean curve, with the individual variation due to continuous unmeasured explanatory variables. Latent class trajectory models allow for distinct groups, presumably representing unmeasured discrete explanatory variables (with unmeasured continuous variables accounted for by a random intercept). Each group of subjects with a given set of measured explanatory variables may fall across several trajectories, but the distribution is modeled with the concomitant variables and need not fall symmetrically around a single curve as in the mixed model.

Importantly, the apparent heterogeneity of cognitive trajectory in these AD subjects is consistent with the current understanding of the heterogeneous nature of underlying neuropathology within individuals receiving a diagnosis of AD. Individuals diagnosed clinically and neuropathologically with AD can still vary substantially in the degree of AD related pathology, i.e. accumulation of $\beta$-amyloid and hyperphosphorylated microtubuleassociated protein tau, that is present on neuropathologic exam. Moreover, individuals diagnosed clinically with $\mathrm{AD}$ express comorbid neuropathologies such as Lewy Body and vascular pathology at high rates. Each of these pathologies may contribute independently to cognitive impairments (Berg et al., 1998).

We found no correlation of the APOE $\varepsilon 4$ allele with any trajectory; this is similar to other studies (Rasmusson et al., 1996; Barnes et al., 2003), though reports of the association of APOE with cognitive decline in the literature vary. Several studies report a positive association of APOE $\varepsilon 4$ with more rapid cognitive decline (Corder et al., 1993; Craft et al., 1998; Martins et al., 2005;O'Hara et al., 2008). Conversely, in an individual growth curve analysis of APOE $\varepsilon 4$-associated cognitive decline in AD, Hoyt et al. (2005) report that although the APOE $\varepsilon 4$ allele is associated with an increased risk of developing $\mathrm{AD}$, subjects with $2 \varepsilon 4$ alleles have a slower clinical course. Kleiman et al. (2006), also report that although APOE $\varepsilon 4$ increased the risk for $\mathrm{AD}$ and decreased the age of disease onset in population studies, it did not significantly influence the rate of disease progression in cognitive or functional domains. Since our cohort 
included only those subjects previously diagnosed with $\mathrm{AD}$, we did not analyze an association of APOE $\varepsilon 4$ with incident AD; however, as with most prior studies, we also found that as the number of APOE $\varepsilon 4$ alleles increased, the age at presentation decreased. One proposed solution to these discrepancies emerges from observations in a longitudinal study analyzing the role of APOE $\varepsilon 4$ in cognitive decline in an elderly population free of dementia at baseline, in which Bretsky et al. (2003), reported that APOE $\varepsilon 4$ was associated with cognitive decline in their population that was most pronounced after 7 years of follow-up. They proposed that the $\varepsilon 4$ allele may either function as a risk factor for cognitive impairment in normal aging, or has effects early in a long prodromal AD trajectory. Further studies in which cognitive trajectories are modeled in subjects with normal cognition who are followed to $\mathrm{AD}$ onset are needed to clarify this ambiguity.

Of the clinical variables studied, the one most strongly related to a trajectory of rapid cognitive decline was greater burden of psychotic symptoms. An association of psychosis with faster cognitive decline has been reported in numerous studies.. In fact, greater cognitive impairment and more rapid cognitive decline is the most consistent correlate of psychosis in AD (Ropacki and Jeste, 2005). To our knowledge, this is the first report to identify trajectories of cognitive decline in AD defined by psychosis burden. Regardless of age and initial MMSE, subjects with psychosis follow trajectories with faster cognitive decline. Because psychosis in AD demonstrates familial aggregation and significant heritability (Hollingworth et al., 2007;Bacanu et al., 2005), our current findings suggest the hypothesis that the more rapid cognitive trajectories associated with cognitive decline may reflect in part the contribution of genetic variations.

The statistical analyses used in this report offer advantages over others. Some prior reports have computed rates of change over short follow-up intervals or over intermittent time intervals, often assuming a linear decline. In the current study, evaluations were performed annually for up to 165 months. Our mixed effects model with latent groups evaluated the variables with a continuous outcome measure of MMSE over time, thus eliminating the need for calculating an estimate of the rate of decline. There is evidence that the rate of cognitive decline may vary at different times in the course of illness, and we confirmed the importance of using a quadratic term in modeling cognitive change over time. Including a quadratic (or higher) term in a trajectory is a standard statistical technique for modeling trajectories that are curvilinear. It is the combination of the terms that describes the shape of the trajectory. If a higher order term is not statistically significant, then only the simpler model is justified (or power is insufficient). If a lower order term is not statistically significant, it is misleading to conclude that there is no linear component to the trajectory; rather the combined equation is the best estimate of the, say, quadratic trajectory. For our best model all but one trajectory (4) show a significant quadratic term (and cubic terms were not significant). We conclude that linear modeling of MMSE trajectories is inadequate, although trajectory 4 may be adequately modeled as a linear decline. Despite the fact that the linear terms in trajectories 1 and 2 are not statistically significant, they do show a curvilinear decline.

Our latent class analysis shows clearly that in all but the lowest ranges of baseline MMSE scores, some subjects deteriorate slowly and others more quickly. The quadratic curve appears to be a reasonable approximation to actual subject curves in these data, but would potentially be less appropriate in datasets with cognitively normal subjects followed to disease onset and death, where subjects may show initial or final areas of little or no change, or more sudden late changes reflecting terminal decline. Of course, there are many events during the course of a long illness, such as delirium, medication toxicity, cerebrovascular insult, or traumatic brain injury that cannot be accurately assayed by any statistical model, further emphasizing the need for careful longitudinal symptom history. 
Several other limitations to the current study exist and deserve comment. The subjects were enrolled from the Alzheimer Disease Research Center, and may not be representative of the general population of elderly subjects with AD. Despite our long follow-up period, some subjects who eventually became psychotic later in the course of their illness may have been missed. Our analyses were also restricted to Caucasian subjects, and may not generalize to subjects with differing socioeconomic or ethnic backgrounds. Methodologically, we used a global measure of cognitive function, the MMSE, as our outcome measure, and different trajectories may have appeared using specific domains of cognitive function.

In conclusion, the present study used a thoughtful statistical approach to determine trajectories of cognitive decline in a large group of phenotypically well-defined $\mathrm{AD}$ subjects. We identified age, initial MMSE, and psychotic symptoms as defining specific courses of cognitive decline, and we provided evidence that trajectory modeling of longitudinal cognitive and behavioral data can resolve phenotypic variation. Future studies using other statistical models and a larger cohort of subjects with cognitive status ranging from normal through $\mathrm{AD}$ will continue to improve our resolution of phenotypes with distinct trajectories. To the extent that specific trajectories reflect the contributions of distinct underlying pathologic processes and/or genetic variations, our approach may enhance the power to detect the association of these neurobiologic mechanisms with cognitive decline and psychosis risk

\section{Acknowledgments}

The authors wish to thank Mr. James Emanuel for formatting the manuscript and the staff of the Alzheimer Disease Research and Clinic at the University of Pittsburgh. We also wish to thank the two anonymous reviewers for many helpful suggestions.

\section{Reference List}

Bacanu SA, Devlin B, Chowdari KV, DeKosky ST, Nimgaonkar VL, Sweet RA. Heritability of psychosis in Alzheimer disease. American Journal of Geriatric Psychiatry 2005;13:624-627. [PubMed: 16009739]

Barnes LL, Wilson RS, Schneider JA, Bienias JL, Evans DA, Bennett DA. Gender, cognitive decline, and risk of AD in older persons. Neurology 2003;60:1777-1781. [PubMed: 12796530]

Berg L, et al. Clinicopathologic studies in cognitively healthy aging and Alzheimer's disease: relation of histologic markers to dementia severity, age, sex, and apolipoprotein E genotype. Archives of Neurology 1998;55:326-335. [PubMed: 9520006]

Bertram K, McQueen MB, Mullin K, Blacker D, Tanzi RE. Systematic Meta-Analysis of Alzheimer Disease Genetic Association Studies: The AlzGene Database. Nature Genetics 2007;39:17-23. [PubMed: 17192785]

Bretsky P, Guralnik JM, Launer L, Albert M, Seeman TE. The role of APOE-epsilon4 in longitudinal cognitive decline: MacArthur Studies of Successful Aging. Neurology 2003;60:1077-1081. [PubMed: 12682309]

Corder EH, et al. Gene dose of apolipoprotein E type 4 allele and the risk of Alzheimer's disease in late onset families. Science 1993;261:921-923. [PubMed: 8346443]

Craft S, et al. Accelerated decline in apolipoprotein E-epsilon4 homozygotes with Alzheimer's disease. Neurology 1998;51:149-153. [PubMed: 9674794]

Curran PJ, Willoughby MT. Implications of latent trajectory models for the study of developmental psychopathology. Development and Psychopathology 2003;15:581-612. [PubMed: 14582933]

Dodge HH, Du Y, Saxton JA, Ganguli M. Cognitive domains and trajectories of functional independence in nondemented elderly persons. Journals of Gerontology Series A Biological Sciences and Medical Sciences 2006;61:1330-1337.

Folstein MF, Folstein SE, McHugh PR. Mini mental state: a practical method for grading the cognitive state of patients for the clinician. Journal of Psychiatric Research 1975;12:189-198. [PubMed: 1202204] 
Gruen B, Leisch F. FlexMix Version 2: finite mixtures with concomitant variables and varying and constant parameters. Journal of Statistical Software 2008;28:1-35.

Healy DG. Case-control studies in the genomic era: a clinician's guide. Lancet Neurology 2006;5:701707. [PubMed: 16857576]

Helgeson VS, Snyder P, Seltman H. Psychological and physical adjustment to breast cancer over 4 years: identifying distinct trajectories of change. Health Psychology 2004;23:3-15. [PubMed: 14756598]

Hollingworth P, et al. Increased familial risk and genomewide significant linkage for Alzheimer's disease with psychosis. American Journal of Medical Genetics Part B: Neuropsychiatric Genetics 2007;144B (7):841-848.

Holmans P, et al. Genome screen for loci influencing age at onset and rate of decline in late onset Alzheimer's disease. American Journal of Medical Genetics Part B: Neuropsychiatric Genetics 2005;135B:24-32.

Hoyt BD, Massman PJ, Schatschneider C, Cooke N, Doody RS. Individual growth curve analysis of APOE epsilon 4-associated cognitive decline in Alzheimer disease. Archives of Neurology 2005;62:454-459. [PubMed: 15767511]

Jones BL, Nagin DS, Roeder K. A SAS procedure based on mixture models for estimating developmental trajectories. Sociological Methods \& Research 2001;29:374-393.

Kamboh MI, Aston CE, Hamman RF. The relationship of APOE polymorphism and cholesteral levels in normoglycemic and diabetic subjects in biethnic population from the San Luis Valley, Colorado. Atherosclerosis 1995;112:145-159. [PubMed: 7772075]

Kleiman T, et al. Apolipoprotein E epsilon4 allele is unrelated to cognitive or functional decline in Alzheimer's disease: retrospective and prospective analysis. Dementia and Geriatric Cognitive Disorders 2006;22:73-82. [PubMed: 16699282]

Leisch E. FlexMix: A General Framework for Finite Mixture Models and Latent Class Regression in R. Journal of Statistical Software 2004;11:1-18.

Lopez OL, Kuller LH, Fitzpatrick A, Ives D, Becker JT, Beauchamp N. Evaluation of dementia in the cardiovascular health cognition study. Neuroepidemiology 2003;22:1-12. [PubMed: 12566948]

Mann UM, Mohr E, Gearing M, Chase TN. Heterogeneity in Alzheimer's disease: progression rate segregated by distinct neuropsychological and cerebral metabolic profiles. Journal of Neurology, Neurosurgery and Psychiatry 1992;55:956-959.

Martins CA, Oulhaj A, de Jager CA, Williams JH. APOE alleles predict the rate of cognitive decline in Alzheimer disease: a nonlinear model. Neurology 2005;65:1888-1893. [PubMed: 16380608]

McKeith IG, et al. Consensus guidelines for the clinical and pathologic diagnosis of dementia with Lewy bodies (DLB): report of the consortium on DLB international workshop. Neurology 1996;47:11131124. [PubMed: 8909416]

McKhann G, Drachman D, Folstein M, Katzman R, Price D, Stadlan EM. Clinical diagnosis of Alzheimer's disease: report of the NINCDS-ADRDA work group under the auspices of Department of Health and Human Services Task Force on Alzheimer's disease. Neurology 1984;34:939-944. [PubMed: 6610841]

O’Hara R, Sommer B, Way N, Kraemer HC, Taylor J, Murphy G. Slower speed-of-processing of cognitive tasks is associated with presence of the apolipoprotein epsilon4 allele. Journal of Psychiatric Research 2008;42:199-204. [PubMed: 17250852]

R Development Core Team. A Language and Environment for Statistical Computing. Vienna, Austria: R Foundation for Statistical Computing; 2007.

Rasmusson DX, Carson KA, Brookmeyer R, Kawas C, Brandt J. Predicting rate of cognitive decline in probable Alzheimer's disease. Brain and Cognition 1996;31:133-147. [PubMed: 8811989]

Ropacki SA, Jeste DV. Epidemiology of and risk factors for psychosis of Alzheimer's disease: a review of 55 studies published from 1990 to 2003. American Journal of Psychiatry 2005;162:2022-2030. [PubMed: 16263838]

Royall DR, Palmer R, Chiodo LK, Polk MJ. Normal rates of cognitive change in successful aging: the freedom house study. Journal of the International Neuropsychological Society 2005;11:899-909. [PubMed: 16519269]

Scarmeas N, et al. Delusions and hallucinations are associated with worse outcome in Alzheimer disease. Archives of Neurology 2005;62:1601-1608. [PubMed: 16216946] 
Schwarz G. Estimating the Dimension of a Model. Annals of Statistics 1978;6:461-464.

Tariot PN, et al. The behavior rating scale for dementia of the Consortium to Establish a Registry for Alzheimer's Disease. American Journal of Psychiatry 1995;152:1349-1357. [PubMed: 7653692]

Tyas SL, et al. Transitions to mild cognitive impairments, dementia, and death: findings from the Nun Study. American Journal of Epidemiology 2007;165:1231-1238. [PubMed: 17431012] 


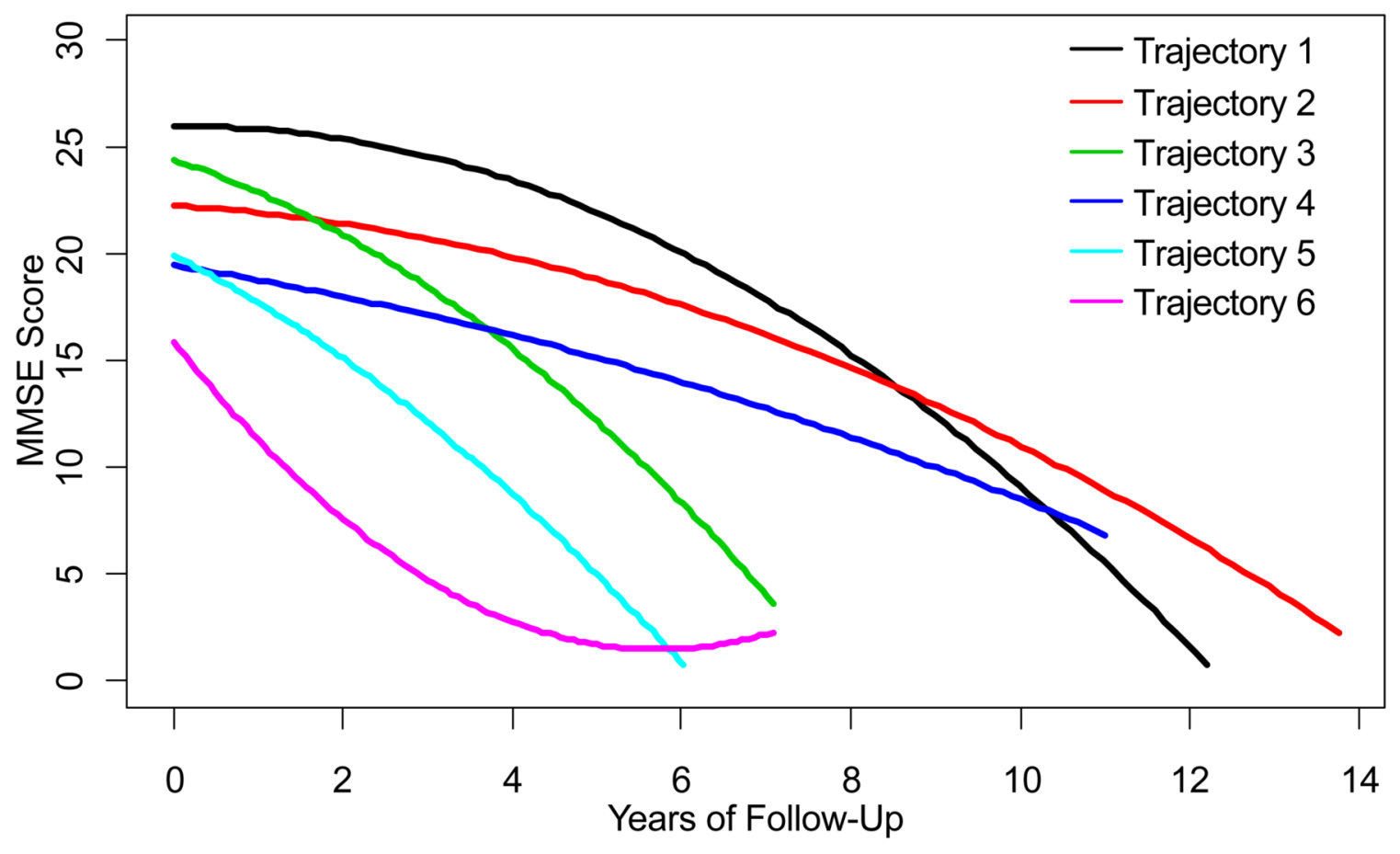

Trajectory Probabilities

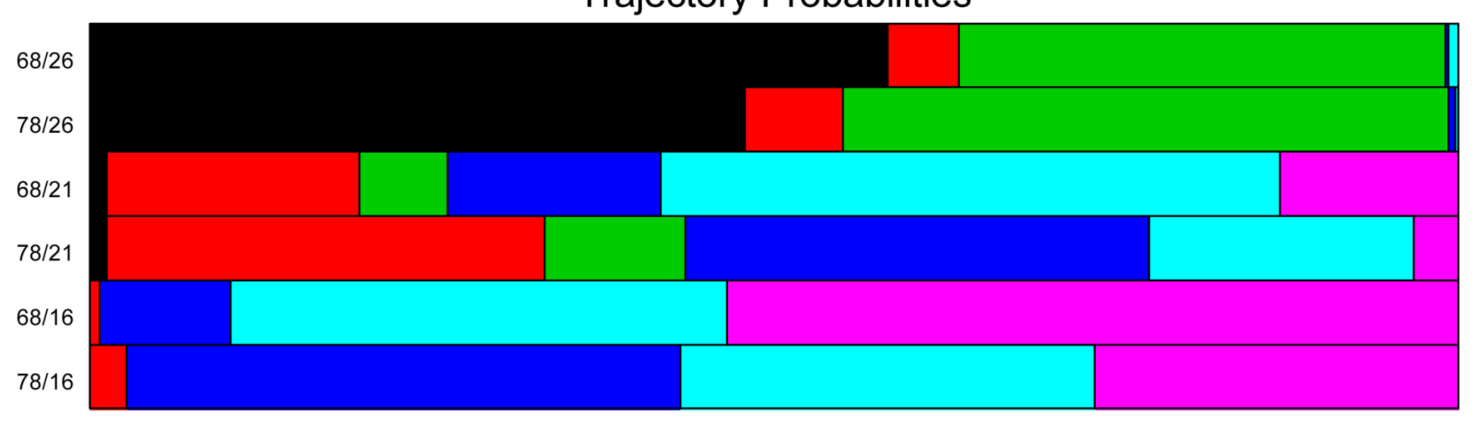

Figure 1.

Cognitive Trajectories of AD subjects and representative concomitant distributions of the trajectories for ages 68 and 78 and initial MMSE scores of 16, 21, and 26. In the top panel, the vertical axis represents MMSE scores; the horizontal axis represents years of follow-up. In the bottom panel the lengths of the bars represent the probabilities of a subject with a particular combination of concomitant variable values falling along the correspondingly colored trajectory. The horizontal extent of each bar represents $100 \%$. 

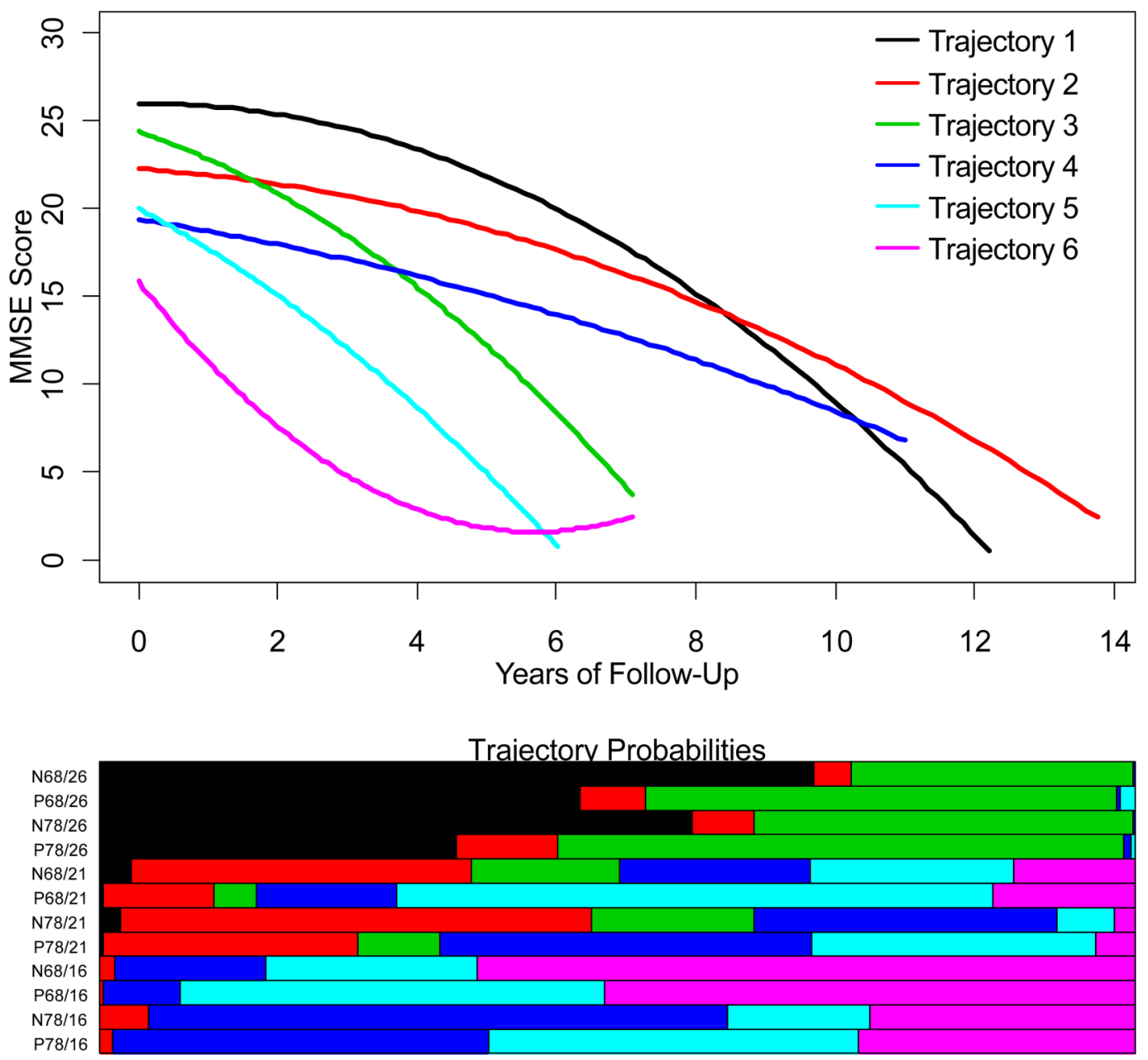

Figure 2.

Cognitive and behavioral trajectories of AD subjects. In the top panel, the vertical axis represents MMSE scores; the horizontal axis represents years of follow-up. The bottom panel is a graphical representation of the probabilities of belonging to a particular trajectory for subjects with particular combinations of initial MMSE, sex and psychosis status. $\mathrm{P}=\mathrm{Psychotic}$; $\mathrm{N}=$ not Psychotic; 26/21/16=initial MMSE, 68/78=age. 


\section{Table 1}

Demographic and Clinical Characteristics

\begin{tabular}{|c|c|}
\hline Clinical Variable & Mean $(\mathrm{SD})$ or $\mathrm{N}(\%)(\mathrm{N}=201)$ \\
\hline $\operatorname{Age}^{l}$ (years) & $73.3(7.97)$ \\
\hline Range & $48.0-87.0$ \\
\hline \multicolumn{2}{|l|}{ Gender } \\
\hline Female & $119(59.2 \%)$ \\
\hline Male & $82(40.8 \%)$ \\
\hline Education 1 & $13.17(2.90)$ \\
\hline Range & $5.0-23.0$ \\
\hline MMSE $^{* 1}$ & $21.0(4.66)$ \\
\hline Range & $6.0-30.0$ \\
\hline \multicolumn{2}{|l|}{ Final Diagnosis } \\
\hline Probable AD & $186.0(92.5 \%)$ \\
\hline Possible AD & $15.0(7.5 \%)$ \\
\hline \multicolumn{2}{|l|}{ APOE $\varepsilon 4$ count } \\
\hline 0 & $83.0(41.5 \%)$ \\
\hline 1 & $91.0(45.5 \%)$ \\
\hline 2 & $26.0(13.0 \%)$ \\
\hline \multicolumn{2}{|l|}{ Psychosis } \\
\hline Never & $84.0(41.8 \%)$ \\
\hline Ever & $117.0(58.2 \%)$ \\
\hline Duration of Follow-Up (months) & $53.1(21.2)$ \\
\hline Range & $19.0-165.0$ \\
\hline
\end{tabular}


Table 2

Parameters of Mixed Model of AD Subjects

\begin{tabular}{lrrrr}
\hline Parameter & Parameter Estimate & Standard Error & t-value & $\mathbf{p}$ \\
\hline Intercept & 20.983 & 0.186 & 113.11 & $<\mathbf{0 . 0 0 1}$ \\
Years in study & -1.280 & 0.132 & -9.66 & $<\mathbf{0 . 0 0 1}$ \\
Years squared & -0.139 & 0.016 & -8.69 & $<\mathbf{0 . 0 0 1}$ \\
MMSE (centered) & 0.849 & 0.029 & 28.94 & $<\mathbf{0 . 0 0 1}$ \\
Age (centered) & 0.035 & 0.017 & 2.05 & $\mathbf{0 . 0 4 2}$ \\
Male sex & 0.736 & 0.276 & 2.66 & $\mathbf{0 . 0 0 8}$ \\
Years in study*age & 0.052 & 0.013 & 3.94 & $<\mathbf{0 . 0 0 1}$ \\
\hline
\end{tabular}

MMSE Mini Mental State Exam 


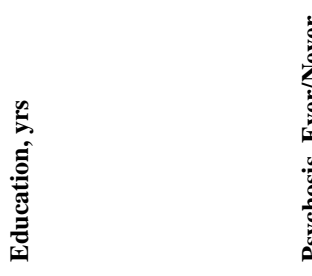

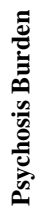




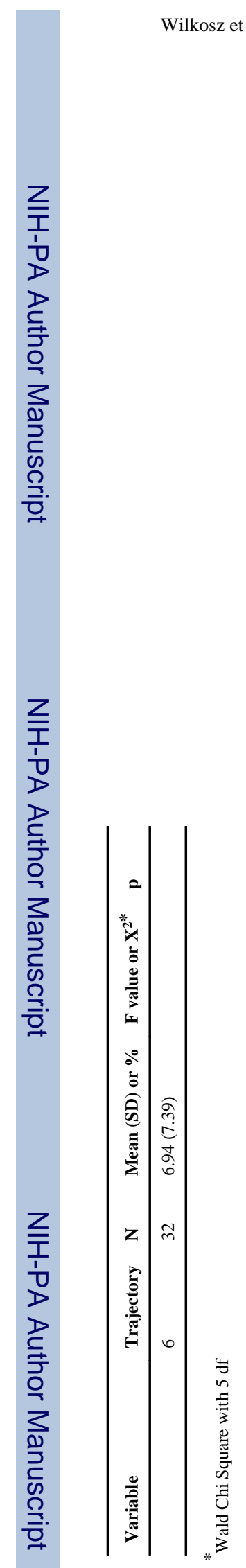

Page 17

Int Psychogeriatr. Author manuscript; available in PMC 2011 March 1. 